\title{
MAPPING THERMAL HABITAT OF ECTOTHERMS BASED ON BEHAVIORAL THERMOREGULATION IN A CONTROLLED THERMAL ENVIRONMENT
}

\author{
Teng Fei ${ }^{\mathrm{a}, \mathrm{b}}$, Andrew Skidmore ${ }^{\mathrm{b}}$, Yaolin Liu ${ }^{\mathrm{a}}$ \\ ${ }^{a}$ School of Resource and Environmental Science, Wuhan University, 129 LuoYuRoad, Wuhan, 430079, P.R. China \\ ${ }^{\mathrm{b}}$ Faculty of Geo-Information Science and Earth Observation (ITC), University of Twente, P.O. Box 217, 7500 AE, Enschede, The \\ Netherlands. \\ feiteng@whu.edu.cn
}

Working Group, Theme or Special Session: VIII/6: Agriculture, Ecosystems and Bio-Diversity

KEY WORDS: thermal habitat, lizard, behavioural thermoregulation, ectotherm

\begin{abstract}
:
Thermal environment is especially important to ectotherm because a lot of physiological functions rely on the body temperature such as thermoregulation. The so-called behavioural thermoregulation function made use of the heterogeneity of the thermal properties within an individual's habitat to sustain the animal's physiological processes. This function links the spatial utilization and distribution of individual ectotherm with the thermal properties of habitat (thermal habitat). In this study we modelled the relationship between the two by a spatial explicit model that simulates the movements of a lizard in a controlled environment. The model incorporates a lizard's transient body temperatures with a cellular automaton algorithm as a way to link the physiology knowledge of the animal with the spatial utilization of its microhabitat. On a larger spatial scale, 'thermal roughness' of the habitat was defined and used to predict the habitat occupancy of the target species. The results showed the habitat occupancy can be modelled by the cellular automaton based algorithm at a smaller scale, and can be modelled by the thermal roughness index at a larger scale.
\end{abstract}

\section{INTRODUCTION}

Among different environmental factors, thermal properties have been used as an important indicator either for the terrestrial animal or for the aquatic animal. Thermal environment is especially important to ectotherm because a lot of physiological functions rely on the body temperature such as thermoregulation (Waldschmidt and Tracy, 1983).

To cope with the wide diversity of thermal qualities of habitat, ectotherms are able to maintain body temperature by continuously shifting their location. The so-called behavioural thermoregulation function made use of the heterogeneity of the thermal properties within an individual's habitat to sustain the animal's physiological processes. This function links the spatial utilization and distribution of individual ectotherm with the thermal properties of habitat (thermal habitat).

Several studies had been focused on the relationship between the thermal properties of land surface and reptile distribution: On scale of microhabitat, we have evidences that the thermal properties of rocks (Schlesinger and Shine, 1994) or shrubs (Kerr et al., 2003) may influence whether reptiles use them as shelters. Reptiles are also sensitive to thermal environment at much larger scales: at continental and global scale, the richness of reptile families is highest at low latitudes (Barnosky et al., 2001). Spellerberg (Spellerberg, 1972) discussed in general the significant relationship between reptile thermoregulatory behavior and distribution. However, Past researches were mainly limited to reptiles' habitat at very large scales, and relatively little is known about how reptiles respond to environmental temperatures at the micro and landscape scales (Fischer and Lindenmayer, 2005).
In this study, we propose to develop an innovative method to map and understand the thermal habitat use of a reptile. The method is based on a spatially explicit dynamic model, which simulate the changing thermal environment and the response of the reptile at the same time.

\section{METHODS}

\subsection{Data collection}

\subsubsection{Thermal Environment}

An experiment was carried out at a reptile zoo "Dierenpark De Oliemeulen" during September, 2008 in Tilburg, the Netherlands. A glass terrarium of size $245 \mathrm{~cm}$ x $120 \mathrm{~cm}$ x $115 \mathrm{~cm}$ was constructed. At the bottom of the terrarium, at least $10 \mathrm{~cm}$ of gravel and sand were mixed to form a flat substrate surface. Photoperiod was maintained at $14 \mathrm{~L}: 10 \mathrm{D}$ with a 100 -Watt heat lamp. An infrared heat lamp of $100 \mathrm{~W}$ provided additional heat input for $5 \mathrm{~h}$ during the middle of the photophase. Temperature sensors $\left(\mathrm{Hobo}^{\mathrm{TM}}\right.$ temperature and relative humidity smart sensors, Onset Computer Co.) were placed at a height of $10 \mathrm{~cm}$ above the ground surface to record the air temperature inside the terrarium. A 4-year-old male Timon lepidus was kept in the terrarium for 10 days before the experiment started, in order that the lizard could acclimatize to the new environment. The lizard was fed on vegetables, crickets, newly born mice, and some fruits such as apple and banana.

\subsubsection{Animal Responses}

Three IRISYS 1011 thermal imagers (each with a resolution of $16 \times 16$ pixels) were mounted in a row at $2 \mathrm{~m}$ above the ground surface of the terrarium, pointing down with a field-of-view 
(FOV) covering a continuous rectangular area of the base of 33 $\mathrm{x} 100 \mathrm{~cm}$. Two webcams (Logitech communication STX with an optical resolution of $640 \times 480$ pixels) were mounted at the same height, with a FOV covering the base. Therefore the dynamics of the thermal environment as well as the animal responses of inside the terrarium were recorded.

\subsection{Model the body temperature}

We aimed to predict accurately the body temperature dynamics of a lizard in any given thermal environment with a physically based model through a rigorous calibration procedure based on Monte Carlo simulation techniques. From a physical perspective, the energy exchange between a lizard and its environment has been described by Porter's models (Porter and Gates, 1969; Porter et al., 1973). This study took this model and made some small adjustments. In summary, the total energy intake of a lizard in a fixed time interval $\left(\Delta Q_{e}\right)$ may be written as the sum of six terms: solar radiation, convective heat flow $\left(Q_{\text {conv }}\right)$, infrared radiation $\left(Q_{\text {longwave }}\right)$, conductive heat flow $\left(Q_{\text {cond }}\right)$, energy gain $\left(Q_{\text {meta }}\right)$ by food intake (metabolism) $\left(Q_{\text {meta }}\right)$, energy loss through respiration/water evaporation $\left(Q_{\text {waterloss }}\right)$.

To further improve the performance of the model and generate realistic parameters, we re-estimated the model parameters using a Monte Carlo simulation. In a preliminary step, the 9dimensional parameter space was sampled over an equally distributed grid. Across each parameter's range, which was assumed to be $\pm 10 \%$ of its reference value, the body temperature model was run at each sample point using the input of the actual thermal environment of the animal experiment, thereby predicting the range in body temperature over time. Meanwhile, the observed lizard body temperature dynamics were recorded, and later compared with the predicted values. Independent observations ( $\mathrm{N}=31$ ) of lizard's body temperature were collected to validate the body temperature model. For a detailed information about the model parameterization please refer to (Fei et al., 2012). Finally, the observations were compared with model simulations and the root mean square error (RMSE) of the temperature prediction was calculated.

\subsection{Predict the thermal habitat occupancy}

The thermal habitat occupancy was predicted through simulating the movement of the lizard. Following a set of transition rules, a simulated lizard performed behavioral thermoregulation as a response to thermal environmental changes. By tracking and aggregate these movements, predictive thermal habitat occupancy maps can be generated. An animal experiment was carried on in a lab and the real habitat usage of a lizard was recorded by cameras. The results were compared with the simulation for the accuracy assessment.

Transition rules form the core of a CA algorithm (Chen et al., 2002). These rules depend on the behavioural traits of the modelled species, their response to thermal landscape dynamics, and their ability to perceive their environment. The assumption made here is that during the day time (from 8:30 to 19:00) the lizard will try to maintain its preferred body temperature $\left(T_{p}\right)$ for as long as possible. When its body temperature falls below (or increases above) $T_{p}$, it will sense the ambient temperature within a certain distance (one cell in our model) and with a chance $P$ it will move to the warmest (or coldest) cell in its vicinity.The transition rules were defined as bellow:

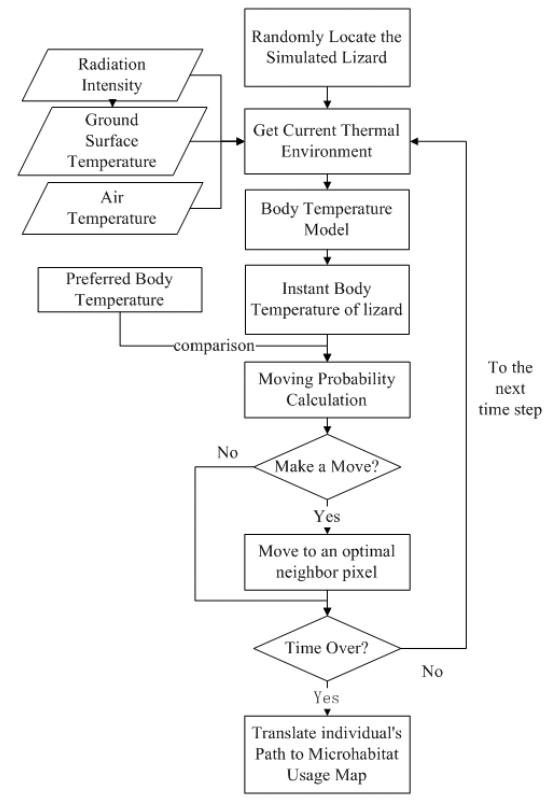

Figure 1. The flowchart of the CA algorithm

The moving probability $\mathrm{P}$ in each step was defined as:

$$
\begin{aligned}
& P=k\left(\left|T_{b}-T_{p}\right|\right), \quad \text { when }\left|T_{b}-T_{p}\right|<\frac{1}{k}, \\
& P=1, \text { when }\left(\left|T_{b}-T_{p}\right| \geq \frac{1}{k}\right),
\end{aligned}
$$

And $\mathrm{K}$ was parameterized from observations of the animal experiment. the detail of the parameterization process can be found at (Fei et al., 2011).

\subsection{Thermal roughness index}

At larger scales, the CA model is computational challenged because of the computational load is proportional to the habitat size as well as the number of individual animals. As an alternative, thermal roughness index is defined to quantified the deviations of a real temperature distribution on a surface from its average value. Thermal roughness index was proposed in this work as a way to predict the occupancy of lizard's thermal habitat. Because of the fact that a reptile regulate its body temperature by shuttling between places with different thermal properties, it make sense that a surface with a more complex thermal conditions is more suitable for the behavioural thermoregulation of reptiles. The thermal roughness index is defined by the arithmetic average of average land surface temperature:

$$
\mathrm{T}_{\mathrm{r}}=\frac{1}{n} \sqrt{\sum_{i=1}^{n}\left(t_{i}-\bar{t}\right)^{2}}
$$

Where $T_{t}$ is the thermal roughness index, $n$ is the number of sample points, $t$ bar is the averaged land surface temperature of the area of interest.The thermal roughness index maps was compared with the simulation results. 


\section{RESULTS AND DISCUSSION}

\subsection{Body temperature modelling}

Independent observations $(\mathrm{N}=31)$ of lizard's body temperature were collected to validate the body temperature model. The observations were compared with model simulations and the root mean square error (RMSE) of the temperature prediction was calculated (figure 2). The result showed that by a biophysical model, the body temperature of the animal can be accurately modeled, and therefore, the model can be integrated into the cellular automaton model to simulate the animal movement.

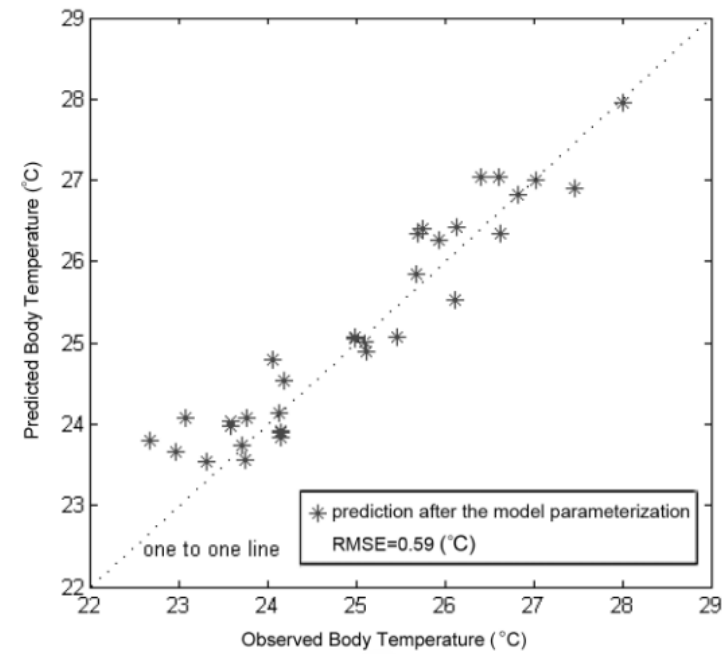

Figure 2. The simulated vs. observed body temperature of a lizard

\subsection{Thermal habitat occupancy}

The integrated model was run to simulate the thermal habitat use by a lizard during a 96-hour experiment. The results of this simulation are illustrated in Figure 5 in a spatially explicit way. The cell colour on the map represents the total time the lizard spent in a particular cell.

For validation, the observatory lizard tracking data was aggregated, classified and compared with the simulation. The accuracy of the simulated thermal habitat use was calculated using a confusion matrix, in which the different levels of occupancy between simulation and actual observation were summarised. The result showed an overall accuracy of $75.7 \%$ (Table 1). It also showed the simulation slightly over-estimated the activity of the lizard.

Table 1. Confusion matrix of the simulated vs. observed microhabitat occupancy (unit: pixel)

\begin{tabular}{ccccc}
\hline & & & \\
Sim. & high & Moderate & low & Row total \\
high & 28 & 16 & 4 & 48 \\
lowerate & 9 & 29 & 32 & 70 \\
& 47 & 78 & 52 & 650 \\
\hline $\begin{array}{c}\text { Column } \\
\text { total }\end{array}$ & 84 & 123 & 56 & $\begin{array}{c}\text { overall } \\
\text { accuracy: } \\
\end{array}$ \\
\hline
\end{tabular}

This result revealed that the thermal environment and the behavioural thermoregulation can be mapped to explain the lizard's microhabitat use. Furthermore, using a cellular automaton algorithm, the spatial pattern of microhabitat occupancy can be simulated solely based on an energy point of view (figure 3).
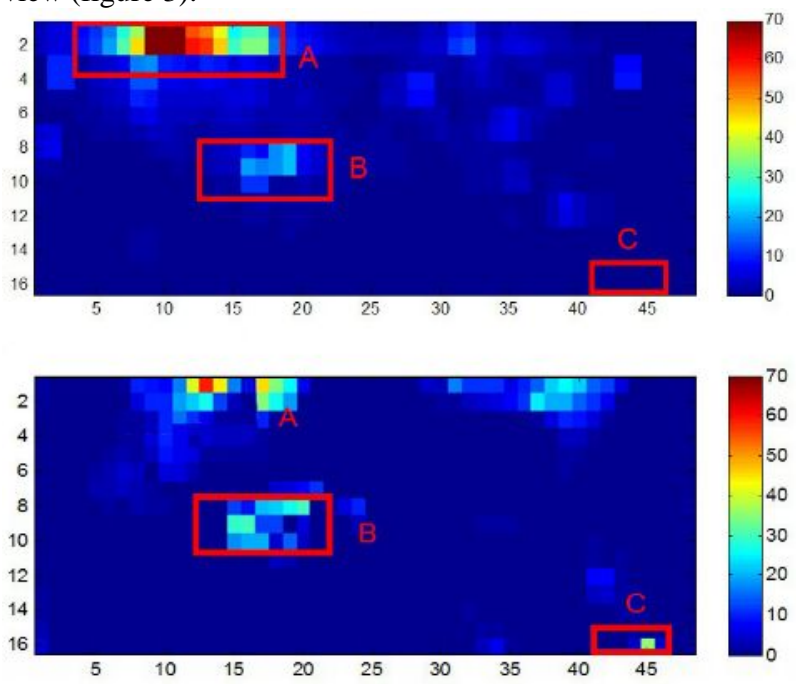

Figure 3 . The simulated vs observed thermal habitat occupancy of a lizard

\subsection{Thermal roughness index}

Although testing the accordance between the habitat occupancies derived from CA model and the thermal roughness index map is an ongoing work, and need rigorous repetitions under different thermal conditions, from the results available, we report that as much as $70 \%$ of lizard habitat occupancy modelled by the CA model can be explained by the thermal roughness index. More results will be reported as we simulate different thermal habitat at different spatial scales in the future.

\section{CONCLUSION}

For the micro-habitat occupancy prediction, an overall accuracy of $75.7 \%$ was obtained. The results suggest that the integrated model of the lizard's body temperature and CA algorithm may accurately predict thermal habitat use by lizards in a controlled environment. For the thermal habitat at larger scales, a newly proposed index: thermal roughness index which has a computational advantage can also be utilized to predict the occupancy of animal thermal habitat as the index provides similar results to the CA model.

\section{References:}

Barnosky, A.D., Hadly, E.A., Maurer, B.A. and Christie, M.I., 2001. Temperate Terrestrial Vertebrate Faunas in North and South America: Interplay of Ecology, Evolution, and Geography with Biodiversity. Conservation Biology, 15(3), pp. 658-674.

Chen, J. et al., 2002. Assessment of the urban development plan of Beijing by using a CA-based urban growth model. Photogrammetric Engineering and Remote Sensing, 68(10), pp. 1063-1071.

Fei, T. et al., 2011. A body temperature model for lizards as estimated from the thermal environment. Journal of Thermal Biology, pp. In press. 
Fei, T. et al., 2012. A body temperature model for lizards as estimated from the thermal environment. Journal of Thermal Biology, 37(1), pp. 56 - 64.

Fischer, J. and Lindenmayer, D.B., 2005. The sensitivity of lizards to elevation: A case study from south-eastern Australia. Diversity and Distributions, 11(3), pp. 225233.

Kerr, G.D., Bull, C.M. and Burzacott, D., 2003. Refuge sites used by the scincid lizard Tiliqua rugosa. Austral Ecology, 28(2), pp. 152-160.

Porter, W.P. and Gates, D.M., 1969. Thermodynamic Equilibria of Animals with Environment. Ecological Monographs, 39(3), pp. 227-244.

Porter, W.P., Mitchell, J.W., Beckman, W.A. and DeWitt, C.B., 1973. Behavioral implications of mechanistic ecology Thermal and behavioral modeling of desert ectotherms and their microenvironment. Oecologia, 13(1), pp. 1-54.

Schlesinger, C.A. and Shine, R., 1994. Choosing a rock: Perspectives of a bush-rock collector and a saxicolous lizard. Biological Conservation, 67(1), pp. 49-56.

Spellerberg, I.F., 1972. Temperature tolerances of Southeast Australian reptiles examined in relation to reptile thermoregulatory behaviour and distribution. Oecologia, 9(1), pp. 23-46.

Waldschmidt, S. and Tracy, C.R., 1983. Interactions between a Lizard and Its Thermal Environment: Implications for Sprint Performance and Space Utilization in the Lizard Uta Stansburiana. Ecology, 64(3), pp. 476-484.

\section{Acknowledgements}

This work was supported in part by the Faculty of International Institute for Geo-Information Science and Earth Observation (ITC), University of Twente, the Netherlands and the national natural science foundation of China (41101411 and 20110491192). 